\title{
CRY FOR HELP: PERCEPTIONS OF YOUNG ADULTS ON SUICIDE FROM NORTHERN PAKISTAN
}

\section{Lubna Ghazal ${ }^{1,2 \varpi}$, David Arthur', Shawkat Hussain $^{3}$, Umerdad Khudadad ${ }^{3,4}$, Gulzar Malik $^{5}$, Zoya Zulfiqar Ali ${ }^{6}$}

\section{ABSTRACT}

OBJECTIVE: To examine the perceptions of suicide among a sample of adolescents from Gilgit-Baltistan and Chitral (GBC), a northern province of Pakistan.

METHODS: A qualitative, descriptive, exploratory design was employed to study the phenomenon by examining secondary data from an asynchronous, online student discussion forum at a University in Karachi. All the participants, originally from GBC, were enrolled from June-December 2019 using a purposive sampling technique based on their experiences of losing a loved one (adolescent sibling or cousin or a friend) because of suicide. The narratives were content analyzed using Creswell framework resulting categories and an overarching theme. Study was approved by ethical committee of university. Pseudonyms were assigned to ensure anonymity and confidentiality of the participants.

RESULTS: Out of 10 participants, 8 were females and 2 were males aged $18-20$ years. Participants shared their perceptions/experiences about suicide among adolescents in GBC, probable root causes of suicide and recommendation/ suggestions in context of GBC. The analysis revealed an overarching theme "cry for help", which was further divided into five categories including: (i) cultural norms; (ii) parents as a whistle for the rat race; (iii) psychological issues; (iv) seeking mental help - a challenge; and ( $v$ ) how can I be helped?

CONCLUSION: Study findings provided insights into factors leading to suicide among young adults in GBC region. The contributing factors highlight the need to develop infrastructure to increase accessibility to healthcare centers, and predominantly access to mental healthcare workers for undertaking risk assessment, and managing the cases promptly is needed.

KEY WORDS: Mental Health (MeSH); Suicide (MeSH); Adolescent (MeSH); Risk Factors (MeSH); Perception (MeSH); Southern Asia (MeSH); Pakistan (MeSH), Gilgit-Baltistan (Non-MeSH); Chitral (Non-MeSH).

THIS ARTICLE MAY BE CITED AS: Ghazal L, Arthur D, Hussain S, Khudadad U, Malik G, Ali ZZ. Cry for help: perceptions of young adults on suicide from northern Pakistan. Khyber Med Univ J 2020;13(2):54-9. https://doi.org/ 10.35845/kmuj.2021.20932.

\section{INTRODUCTION}

S uicide is a serious public health issue. In 2012, 804,000 deaths related to suicide were reported around the world, with one attempt every 40 seconds.' In ages 15 to 29 years, it is the second leading cause of death globally', with the age-standardized incidence rate of I I 4 per I00,000 populations. The rates are higher in males compared to females (I5 per 100,000 vs. 8 per 100,000$){ }^{2}$ A scoping review of six South Asian countries revealed a varied occurrence of adolescent suicide ranging from 0.43 / 100,000 to 331 per $100,000 .^{3}$ However, in some developing countries, such as
Pakistan, no systematic suicide surveillance systems are in place, while social and mainstream media remain the major sources of information. This impedes the understanding of the extent and reasons contributing to suicide in Pakistan and hinders the ability to make comparisons. ${ }^{3}$

There is a scarcity of information available on suicide in Pakistan. In Pakistan, the reported increase in the number of suicide attempts, particularly in the northern region of Gilgit Baltistan and Chitral, ${ }^{4}$ has prompted the need for creating and strengthening surveillance systems to collect and disseminate data imperative for immediate action. ${ }^{5,6}$
I: Faculty of Health Sciences, Aga Khan University, Karachi, Pakistan.

2: Continued Professional Education Department, Mukhtar A. Sheikh Hospital, Multan, Pakistan.

3: Karwan-e-Hayat, Psychiatric Rehabilitation Facility, Karachi, Pakistan.

4: Department of Emergency Medicine, Agha Khan University, Karachi, Pakistan.

5: Latrobe University, Australia.

6: University of Toronto, Canada. Contact \# + 92-336-1858653 Email凹: lubna.ghazal@aku.edu Date Submitted: October 14,2020 Date Revised: May 25, 2021 Date Accepted: June 05, 202I

The situation in Gilgit-Baltistan and Chitral

Gilgit-Baltistan and Chitral which is an important part of northern Pakistan, sharing borders with Afghanistan and China and comprising eight mountainous valleys with an estimated population of I.8 million. The capital city of Gilgit (population 216,760) is considered an area deprived of healthcare facilities and skilled professionals, in particular mental health professionals, very few of whom are accessible to identify and address the mental health issues of the community. ${ }^{7}$ The incidence of adolescent suicide is growing and is usually reported, through the local newspapers, with few refereed studies published. A study in the context of Gilgit Baltistan revealed that 49 females committed suicide in Ghizer, a valley of Gilgit Baltistan between the years 20002004. Among these, adolescent girls and young females aged 15-24 years, rates were reported to be high as $61.07 /$ 100,000 per year. ${ }^{8}$ These figures are considerably higher than those in other parts of Pakistan. The underlying causes have not yet been fully studied and requires studies to establish the causes, patterns and preventive approaches. However, a few recent studies investigating suicide attempts among youth in Northern areas of Pakistan concluded that the causes mostly relate to their socio-cultural situation. While exploring 
the perceptions of youth (between ages of 15 to 25 years) residing in Hunza district of Gilgit, the researchers revealed rapid modernization, relationship challenges and parental approaches as major causes of suicide among young adults. ${ }^{7-9}$ Additionally, a study found that social (46\%), cultural (35\%) and psychological (19\%) factors were underlying causes of suicide attempts in the context of Gilgit Baltistan. ${ }^{10}$ These studies have highlighted the need to further explore underlying cultural and sociological issues leading to youth suicide in the context of Gilgit Baltistan and Chitral. A deeper understanding of the of the phenomenon in the context of unique culture in northern Pakistan is vital and will assist in creating comprehensive and valid suicide prevention strategies both at national and international levels.

\section{METHODS}

This study explored the perceptions of suicide among adolescents from Gilgit Baltistan and Chitral, the northern region of Pakistan. A qualitative, descriptive exploratory design was employed. Since the study aimed to understand suicide phenomena among young adults from Gilgit Baltistan and Chitral, the northern region of Pakistan, therefore a descriptive qualitative approach was considered appropriate.

This study included young adults aged I820 years, enrolled in an associate degree at a private University in Karachi, Pakistan. Participants who were originally from Gilgit Baltistan and Chitral region were purposely and conveniently selected. The selection was also based on their experiences of losing a loved one either an adolescent sibling or cousin or a friend because of suicide. Purposive sampling allows a researcher to recruit individuals who have experienced the phenomenon closely and are able to provide rich information about the phenomenon of interest.

Data was collected from an online focused group discussion. A semistructured online discussion guide was developed based on previous studies conducted about the phenomenon in the context of Pakistan. Following the principles of focused group discussion, the facilitator generated conversation on the followings:

- Perceptions/experiences about suicide among adolescents in Gilgit Baltistan and Chitral, northern Pakistan.

- Root causes of suicide among adolescents in Gilgit Baltistan and Chitral, northern region of Pakistan.

- Recommendation/suggestions in Gilgit Baltistan and Chitral context.

An online discussion forum was created by primary investigator. This discussion forum was a closed group with a focused group discussion activity (asynchronous), whereby participants were allowed to share their experiences and perceptions in writing related to the phenomenon of study. The above pointers were posted on the forum and participants were given opportunity to express their views and contribute to the discussion. As an advantage of an asynchronous online discussion forum participants were able to offer their views freely on the forum at their time of convenience. The discussion was continued (up to two weeks) until the recurrent themes were noted. Data saturation was used as a parameter to hold data collection when there was repetition of information or no new information was obtainable.

Permission to analyze secondary data from the online discussion forum was sought from the ethics committee of the university (ERC 2018-0482-934). The access of the data was in the custody of primary investigator and research team. Consent from the participants was obtained over email. Transcripts were generated and analyzed using content analysis steps by Creswell, 2015, whereby important words, phrases, or ideas were coded. Similar codes were then identified and compared, and aggregated to form categories, sub-categories and subsequent themes. Pseudonyms were assigned to ensure anonymity and confidentiality of the participants.

\section{RESULTS}

Out of 10 participants, 8 were females and 2 were males aged $18-20$ years. The overarching theme "Cry for help" emerged from the analysis of the data as many participants have used this term to describe their perception related to suicidal thoughts, ideation and cues before someone attempt suicide. This theme was further explored into five categories: cultural norms; parents as a whistle for the rat race; psychological issues; seeking mental health - a challenge; and how can I be helped? Figure I illustrates the visual presentation of the categories.

\section{Cultural norms}

Culture sets the foundation of the core values and behaviors within individuals. Within the culture of Gilgit Baltistan and Chitral, it is common practice for young women to be 'married off' to men, based on the preference of their parents. Once a girl enters her adolescent years, she is forced to put aside her childhood freedoms and wishes, such as getting an education and being independent. Participant I captured the essence of this in the testimony: ....this is in our (north Pakistan) culture that girls are not allowed to consent for their wedding, instead they are told to marry a person arranged for marriage by their parents (Participant I).

The norm of arranged marriages in this community was discussed by a number of participants. The lack of freedom of choice and the need to suppress desire was highlighted as a feature of the life of adolescent girls, and the conflict between their wishes and the parental expectation generated much discussion, with one participant suggesting this was a cause of suicide in some girls: ... most of the times, parents force their children into arranged marriages; parents decide and choose the partners, which becomes one of the reasons for suicide [among adolescents] (Participant 2).

Respondents also felt that females rather than males are forced into arranged marriages. Another participant shared an experience of a friend whom she considered a victim of the cultural norm of arranged marriages: ... one day her dad called and forced her to get married with her cousin, she didn't want to marry him, and she ended her life (Participant 3).

Most of the participants felt that getting married at a young age and experiencing rapid and stressful transition from being an adolescent to a married woman, req- 


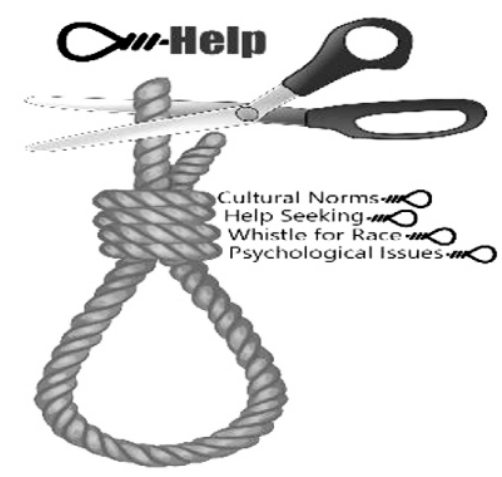

Figure I: Visual Presentation of the categories

uiring them to care for their new family beyond their capacity, was shocking. For teenaged married women, these expectations were reported as intense and difficult to cope with. For example:

Women in Gilgit Baltistan have a heavier workload than men, in running a house. They wake up early in the morning because they have to do all the domestic work like cooking food, washing clothes, taking care of their children and pet animals, pastures and working in fields (Participant 2).

Most of the participants were clear in their opinions that forcing decisions on women is a common practice in Gilgit Baltistan and Chitral, which devalues the opinions and decisions of women and creates dependency on their fathers at an early age. This dependency in turn is transferred onto the husband, which reinforces the lower status of women. Young women are forced to subdue urges that can potentially harm the reputation of their families. They are taught to restrain from expressing their opinions in order to allow men and elders to appear forthright in decision making:

If a girl likes to make her point even though her points are valid and strong, we would expect that, others (neighbors, society) would say 'her parents never taught her not to cross question elder or dominant people'. If younger people try to correct elders, a common response is "this baby of a few days is trying to teach me (Participant 3).

Overall, the participants explained how the lives of females in Gilgit Baltistan and Chitral were constricted, and reflective of a male dominant society. Men have the most power to make decisions, as a participant touched on by saying, "It's a male dominant society and females don't have freedom to speak e.g. a man can divorce a woman at any time in our society and go for a second marriage" (Participant 2). Young women on the other hand have little say and hence become stressed, which becomes a contributing factor to their deaths by suicide.

\section{Parents as a whistle for the rat race}

There was a perception that some parents place high expectations on their children with little support and feedback. Parents punish their children harshly and use their authority to enforce demands. Specifically, in this context, children are expected to demonstrate high academic performance and are punished if the expectations are not met. One participant commented:

In Gilgit Baltistan and Chitral, parents force their children to study with full focus and get full marks in their examinations and make themselves feel proud. If a child is not interested in studies, and cannot get good marks and fails, the child becomes afraid of telling his parents about his results because he knows his parents won't listen to him. He chooses to run away from this situation, which leaves little choice but suicide (Participant 5).

Furthermore, another participant touched upon the consequences some children may face if they fail to meet the high academic demands of their parents by saying: Parents appreciate those children who gain good marks and for those who cannot, parents always complain and compare them with their friends or another child which can cause early stages of aggression and depression in a child (Participant 6).

Another poor parenting style was highlighted wherein children receive minimal nurturing, guidance, and limited attention. One explained how in certain situations children are neglected leading to mental health issues: .... a person or a child getting rejected or neglected by his own parents, his own family members, leads to feelings of hurt. In a family, if there are five or six siblings and parents are not able to give equal attention to all children, then those who are neglected feel like they are the burden to their parents (Participant 5).

It was clear that children in their communities are afraid to express their emotions to their parents, due to a fear of receiving a violent response or having their thoughts disregarded. This forces kids to turn to their friends and others for personal support, which further reinforces the distance between them and their parents as a participant shared: Poor relationship among parents and children is a contributing factor towards committing suicide. In these situations, children tend to share their personal secrets with their friends. If there is breakup in the friendship, the fear of having a secret disclosed can become a cause of suicide. A 15- yearold girl committed suicide because she had shared personnel secrets with her friend. This friend shared the secret with others, and the girl, she committed suicide in fear of her secrets being spread and her family finding out all about her (Participant I).

\section{Psychological issues}

Depression and ongoing emotions of extreme sadness and helplessness can lead to suicide. Many of the participants made comments about the relationship between psychological disorders and suicide. A strong feeling of sadness invoked through depression was explained to be a driving force for individuals who died by suicide. Some believed that the presence of other emotions, such as, loneliness, depression and feelings of being useless could potentially lead to suicide as articulated by a participant: What I believe is that amongst all the factors, depression is the most important factor which connects to other things. For example loneliness, inferiority complex, generation gap, can be linked with depression (Participant 7).

Another participant explained how intense emotions, such as loneliness, invoked by depression can cause individuals to feel rejected and trapped:

The people who commit suicide are the ones who have family and friends but still feel alone... These reasons are causes of stress and depression and they are trying to run away from these situations. The worst feeling is getting rejected by their peers or family which makes them feel lonely (Participant 8).

One of the participants explained multiple reasons as to why they believe suicide 
occurs in relation to psychological issues: Suicide is a multi-faceted issue, sometimes an act of heroism, an act of selfishness, and sometimes a brave choice in the face of pain. That's what a person thinks before committing suicide. I think the main cause of suicide is depression (Participant 9).

Another participant articulated the idea that individuals who face suicidal thoughts feel trapped and hopeless, highlighting and connecting the strong emotions that are present: People who commit suicide usually think that suicide is the only solution to their hopelessness, emptiness, guilt and rejection (participant I0).

It seems that adolescents in Gilgit Baltistan and Chitral are in a chaotic situation where they are expected to meet both household and societal expectations which leaves little room for their personal feelings. Divesting children from their personal feelings leads to depression and despair, resulted in feelings of being misunderstood and believing that suicide is the only way out. Suicide becomes a means to help them overcome numerous problems in life.

Seeking mental health help - a challenge

Study participants highlighted the lack of resources addressing mental health issues in Gilgit Baltistan and Chitral. Health care services are limited with few mental health professionals readily available to provide care. One participant reflected: In Gilgit Baltistan, we lack health services and professionals. Eight years ago, I had seen a corpse of women, she committed suicide because she was not mentally stable, however there was no one around to help her professionally (participant 9).

Individuals have difficultly accessing help and when there is no professional help available, suicide is an obvious outcome, as highlighted by another participant: "In the entire Hunza region, we cannot find a psychologist which ultimately increases the risk of long-term depression and suicide" (Participant 7).

\section{How can I be helped?}

Participants of the study provided possible solutions for preventing suicide, for example, by strategic planning at an individual, family and community levels. At an individual level, adolescents can be assisted by showing empathy and compassion towards those who possess suicidal ideations. Individuals with emotional and behavioral difficulties should be assisted by providing emotional support. One participant expressed her views on preventing suicide at the individual level:

Using positive coping strategies, such as peer support, avoid using drugs or alcohol and taking help of the counselor prevents suicide (Participant 5).

Another participant voiced: "Individual problem solving and decision-making skills have a role in the prevention of suicide" (Participant I0).

Highlighting community support in preventing suicide, another articulated: "Everyone should be encouraged to support individuals with suicidal thoughts instead of bullying them" (Participant 8). Strong family support plays a significant, positive role in the prevention of adolescent suicide. The family provides emotional support to the child, which is required during the most challenging times, as shared by a participant: "Parents should give good guidance to their children and have friendly relationship within a family...children should be supported by their parents to share their problems" (Participant 3).

At the community level in Gilgit Baltistan and Chitral, the stakeholders of the community were seen to hold responsibility for taking measures to prevent adolescent suicide by providing social support to vulnerable individuals and fighting discrimination against adolescents based on the academic performance or societal expectations of the female gender: Local community programs and networks should be established to prevent suicide. It is the responsibility of the government, media, and law enforcement agencies to take measures (Participant 4).

We need to have social institutions at the community level and government institutions as well to help people with suicidal thoughts (Participant 6).

In Gilgit Baltistan and Chitral, there are limited numbers of mental health professionals available to help adolescents with psychological issues, and there is no mental health facility where people can seek help and treatment for mental illnesses. A couple of participants mentioned: "Everyone should have access to professional health workers” (Participant 9). “...healthcare system should focus on mental health particularly in Gilgit Baltistan" (Participant 8).

\section{DISCUSSION}

Suicide is a complex issue requiring better understanding of the influencing factors and its prevalence. In this study, each participant provided insights and explanations that create a unique picture of suicide in Gilgit Baltistan and Chitral, northern region of Pakistan. The environmental and geographical factors intertwined with the cultural factors coincide with the literature that suggests suicide rates are influenced by the social and economic characteristics and the geography of different areas. ${ }^{10,11}$

The findings also revealed that culturally, women are restricted in freedom of expression and thought. They are forced to live in accordance with what is expected from their gender and to abide by the guidelines provided from those 'superior' to them. Within households, men are the breadwinners while women provide care for the families. The findings from this sample revealed specific cultural patterns that impact suicidal behavior, which are consistent with the findings of previous research conducted in this region. ${ }^{10,11}$ In addition, it emerged that it is common for young women to be married off to men of the parents' choice - more specifically their fathers' choice. A father will look for a man who can bring wealth and respect to his family's reputation. Young women have little say in whom they marry, as they are required to fulfill the wishes of their parents out of respect. This suppression essentially offers limited opportunities and avenues for women to express their opinions, wishes and desires, and this was seen to lead to low self-esteem and depression. These findings corroborate with evidence from both international and national literature. ${ }^{12,13}$

Family dynamics is a highly influential factor in the mental well-being of an individual. From birth, family members 
provide guidance, and their values and beliefs construct the intrinsic nature and shape overall development of a child. If parenting is flawed, then the upbringing of the child and their development is largely affected. This emerged from the data as the 'parenting styles' sub-category. Participants raised issues around two types of parenting styles- authoritarian and uninvolved. In the former, parents enforce rules with little guidance and support - the feelings of the child are ignored, and authority of the parent is absolute. When parents' demands are not met, the consequences are harsh. Authoritarian parenting styles are said to increase the risk of suicidal ideation two-fold and the risk of deliberate selfharm three-fold in adolescents. ${ }^{14}$ In uninvolved parenting, guidelines are not provided and there is a lack of attention to the child's needs and demands, and there is a significant association between uninvolved parenting and suicide attempts among adolescents, which suggests increases the risk of suicide attempts by a factor of $1.5 .^{15}$ The child experiencing this kind of parenting style feels neglected and unwanted by their parents, which affects their self-esteem. Studies suggest that in both kinds of parenting styles, the child becomes distant from their parents due to lack of communication and disconnect. They suffer from social isolation and essentially become susceptible to mental health issues. ${ }^{16}$ Interestingly, the current study revealed that the father figure was reported to adopt the authoritarian parenting style while mother was seen more as uninvolved due to her heavy workload and lack of freedom of speech and involvement in decision making in the child's life. This combination of parenting for a child leaves no space for an adolescent seeking support in their cry for help.

Findings also highlight that because of the many mountains and valleys, the northern region is surrounded by; it is difficult for individuals to travel the distances required to access healthcare, education and recreational resources, which are crucial building blocks to strong communities and societies. Healthcare facilities, clinics and hospitals are scarce, also the inaccessibility to mental health providers has been shown to be associated with higher suicide rates in this region. ${ }^{17}$ It is evident from this study that psychological issues such as hopelessness, feelings of rejection and depression resulting from poor parenting styles, lack of resources and disparaging cultural norms contribute to suicide in Gilgit Baltistan and Chitral.

Several studies have reported that depression, anxiety and ineffective coping from stress are considered the major factors leading to self-harm ${ }^{\text {17-19 }}$ particularly, hopelessness increases the risk of suicide among individuals living in low sociodemographic conditions. ${ }^{20,21}$ The participants' perceptions of suicide in Gilgit Baltistan and Chitral were congruent with the findings from reputable studies and although it was a small sample it highlighted a clear theme of a 'cry for help' which offers valuable insight into the extend of the issue and provides a platform for further research and practical interventions.

\section{LIMITATIONS}

The limitations of this qualitative, small sample study, preclude generalization of the findings beyond the group in the study, and clearly there is a need for studies on larger samples. Additionally, immediate testing of interventions for vulnerable groups ${ }^{22,23}$ for instance in Gilgit Baltistan and Chitral is recommended.

\section{CONCLUSION AND RECOMMENDATIONS}

The findings from this study, create awareness of the influencing factors including environmental, geographical along with the cultural influence results in higher rates of suicide in Gilgit Baltistan and Chitral region. These highlighted the need for an infrastructure to support those who suffer with mental illnesses and are susceptible to suicide attempts. Accessibility to healthcare centers and to mental health professionals is essential for conducting risk assessment, recognizing the signs of suicide and managing cases promptly. Given that suicide is increasing throughout Pakistan, it is an absolute priority to develop suicide prevention plans at the national level, particularly in the most affected areas like Gilgit Baltistan and Chitral, northern Pakistan. Multi-faceted strategies focusing on risk factors and underlying causes should be addressed through media, education, law enforcement agencies and courts.

Particular attention needs to be given to youth and adolescents who are considered as the most vulnerable group. Support, assistance, and attention are needed to allow them to express their views freely, empower them with stress management skills and communication tools to face challenging situations of life. Raising awareness of the services available to seek prompt help needs to be integrated through schools/colleges and universities. There is also a need to educate and empower parents about this alarming situation and how parents can play a critical role in bringing this serious issue under control. Suicide is a public health crisis and requires an urgent call to formulate a national mental health policy and interventions to manage this highly pressing issues. ${ }^{24}$

\section{ACKNOWLEDGMENT}

Authors are thankful to study participants for sharing their valuable insights.

\section{REFERENCES}

I. World Health Organization (WHO). Suicide in the world: global health estimates. 2019. (Accessed on: November 28, 2020). Available from URL: https://www.who.int/publica tions/i/item/suicide-in-the-world

2. Bachmann, S. Epidemiology of suicide and the psychiatric perspective. Int J Environ Res Public Health, 2018; 15 (7): 14-25. https://doi.org/10.3390/ ijerph I 507 I 425

3. Jordans MJ, Kaufman A, Brenman NF, Adhikari RP, Luitel NP, Tol WA, et al. Suicide in South Asia: a scoping review. BMC Psychiatry 20 I4; I4(I): 358-64. https://doi.org/10.1/86/ sl2888-0|4-0358-9

4. Khan MM, Reza H. The pattern of suicide in Pakistan. Crisis 2000;2I ( I): 3I-5. https://doi.org/ 10.1027//02275910.21 .1 .31

5. Anjum A, Ali TS, Pradhan NA, Khan $M$, Karmaliani R. Perceptions of stakeholders about the role of health system in suicide prevention in Ghizer, Gilgit-Baltistan, Pakistan. 
BMC Public Health 2020;20:991. https://doi.org/I0.1 |86/s I 2889020-0908I-x

6. Dad AM. Why commit suicide, when we have one life to live. Pamir Times, March 6, 2018. [Accessed on October 0I, 2020]. Available from URL: https://pamirtimes.net/ 2018/03/06/why-to-commit-suicidewhen-we-will-live-only-once/

7. Rahnuma B, Fangtong J, Khan MA, Saddique K, Ahmad I. Causes of Suicide in Gilgit-Baltistan Region. Eur Acad Res 20I 7;8(5):4040-5I.

8. Khan MM, Ahmed A, Khan SR. Female suicide rates in Ghizer, Pakistan. Suicide Life Threat Behav 2009;39(2):227-30. https://doi.org/ |0.152 I/suli.2009.39.2.227

9. Sher S, Dinar H. Ethnography of suicide: a tale of female suicides in district Ghizer, Gilgit Baltistan. J Soc Sci 20I0;I (6):207-I0.

10. Muneeba S. A Qualitative Analysis of Suicides Committed by the Students in Pakistan. Pak J Med Res 2019;58:35-40.

II. Agerbo E, Sterne J, Gunnell D. Combining individual and ecological data to determine compositional and contextual socio-economic risk factors for suicide. Soc Sci Med 2007;64(2):45I-6I. https://doi.org/ 10.1016/j.socscimed.2006.08.043

12. Shain B. Suicide and suicide attempts in adolescents. Pediatrics 2016;138 (1):e2016I420. https://doi.org/ 10.1542/peds.2016-I420
I3. Naveed S, Qadir TF, Afzaal T, Waqas A. Suicide and Its Legal Implications in Pakistan: A Literature Review. Cureus 2017;9(9):el665. DOI:10.7759/ cureus. 1665

14. Donath C, Graessel E, Baier D, Bleich $\mathrm{S}$, Hillemacher T. Is parenting style a predictor of suicide attempts in a representative sample of adolescents? BMC Pediatrics 20I4; I4(I):II3. https://doi.org/I0.1 |86/|47|-243। 14-113

15. Yousefia S, Far AS, Abdolahian E. Parenting stress and parenting styles in mothers of ADHD with mothers of normal children. Procedia Soc Behav Sci 20 I I;30: 1666-7I. https://doi.org/ I0.1016/j.sbspro.201।.10.323

16. CherryK. The effects of authoritarian parenting on children. Accessed on: January 23, 2019. Available from URL: https://www.verywellmind. com/what-is-authoritarianparenting-2794955C, 2018

17. Gould M, Munfakh J, Kleinman M, Lake A. National Suicide Prevention Lifeline: Enhancing Mental Health Care for Suicidal Individuals and Other People in Crisis. Suicide Life Threat Behav 2012,42(I):22-35. https://doi.org//0.1। II/j. I 943278X.2011.00068.x

18. Ibrahim N, Amit N, Suen MWY. Psychological Factors as Predictors of Suicidal Ideation among Adolescents in Malaysia. PLoS One, 2014;9(I0): el 10670. https://doi.org//0.137I/ journal.pone.01 10670.
19. Shekhani SS, Perveen S, Akbar K, Bachani S, Khan MM. Suicide and deliberate self-harm in Pakistan: a scoping review. BMC Psychiatry $2018 ; \mid$ 8(I):44. https://doi.org/ |0.| |86/s | 2888-0|7-1586-6

20. Smischney TM, Chrisler A, Villarruel FA. Risk Factors for Adolescent Suicide. Int J Med 20 I 4; I08(I0):765-80.

2I. Huver RME, Otten R, de Vries H, Engels RCME. Personality and parenting style in parents of adolescents. J Adolesc 2010;33(3): 395-402. https://doi.org/10.1016/ j.adolescence.2009.07.012

22. World Health Organization (WHO). National suicide prevention strategies: progress, examples and indicators, 2018. Accessed on: January 23, 2019. Available from URL: https://www.who.int/ mental_health/suicide-prevention/ national_strategies_2019/en/

23. Wardle H, McManus S. Suicidality and gambling among young adults in Great Britain: results from a crosssectional online survey. Lancet Public Health 202l;6(I):e39-49. https://doi.org/10.1016/S24682667(20)30232-2

24. Khan MM, Mahmud S, Karim MS, Zaman M, Prince M. Casecontrol study of suicide in Karachi, Pakistan. $\mathrm{Br} J$ Psychiatry 2008; 193(5):402-5. https://doi.org/10.1 192/bjp.bp. 107.0 42069

\section{AUTHOR'S CONTRIBUTION}

All authors (LG, DA, SH, UH, GM, ZZA) have made substantial contributions to the manuscript conception and study design, acquisition analysis and interpretation of data, drafting the manuscript, critical review, approval of final version to be published.

Authors agree to be accountable for all aspects of the work in ensuring that questions related to the accuracy or integrity of any part of the work are appropriately investigated and resolved.

CONFLICT OF INTEREST
Authors declared no conflict of interest
GRANT SUPPORT AND FINANCIAL DISCLOSURE
Authors have declared no specific grant for this research from any
funding agency in the public, commercial or not-for-profit sectors
DATA SHARING STATEMENT
The data that support the findings of this study are available on request from the corresponding author. The data are not publicly available due to privacy or ethical restrictions.

This is an Open Access article distributed under the terms of the Creative Commons Attribution-Non Commercial 2.0 Generic License. 